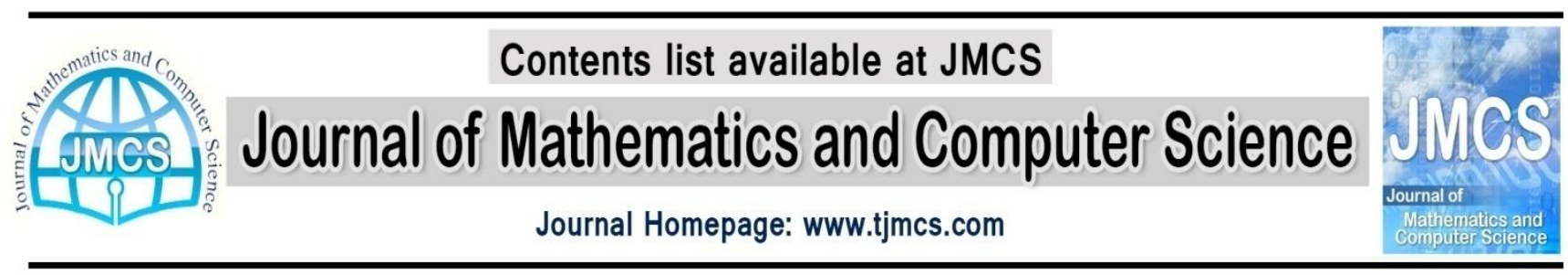

\title{
A method for calculating interval linear system
}

\author{
Shohreh Abolmasoumi * , Majid Alavi \\ Department of Mathematics, Arak branch, Islamic Azad University, Arak, Iran \\ sh.masoumi55@yahoo.com \\ m-alavi@iau-arak.ac.ir
}

\begin{abstract}
Article history:
Received June 2013

Accepted July 2013

Available online July 2013
\end{abstract}

\begin{abstract}
In this paper we represent an efficient algorithm for finding the interval solution for the interval linear system. This algorithm applies the optimization problem based on gradient vector in order to obtain the lower bound and upper bound of the interval solution.
\end{abstract}

Key word: Interval number, Interval linear system, Interval matrix, Cramer's rule, Gradient vector, Multivariate function.

\section{1: Introduction}

When the calculation of a square and nonsingular matrix whose entries are real numbers is concerned, one of the computing methods is that one writes it down in the form of linear equation system composing of the product of $n \times n$ by $n \times 1$ vector where the former denotes the coefficients and latter represents the unknowns. In interval arithmetic direct method have been introduced e.g. the interval Gauss algorithm [2] Computation of algebraic solution to interval system via system of coordinates, Scientific computing, Validated numerics, Interval methods [9]. The interval linear equation system and interval arithmetic proposed by [14] are employed in order to compute interval linear system. Also, the definitions of Cramer's rule, which are fundamental parts of, gradient vector and multivariate function is introduced. The organization of the paper is follows. In Section 2, the definitions related to interval number, and

* Corresponding author. 
interval matrix is given briefly. Then interval arithmetic and interval linear equation systems we clarify Cramer's rule for solving an interval liner system. In Section 3 includes some new definitions pertinent to calculating the interval linear system matrix. An example is given in Section 4. Final section is a conclusion.

\section{2-1: Preliminary.}

Definition (2-1-1): Really that the closed interval denoted by $[a, b]$ is the set of real numbers given by

$$
[a, b]=\{x \in \mathcal{R}: a \leq x \leq b\}
$$

Although various other types of intervals (open, half-open) appear throughout mathematics our work well center primarily on closed intervals. In this paper, the term interval will mean closed interval. We will adopt the convention of denoting intervals and their endpoints by capital letters. The left and right endpoints of an interval $A$ will be denoted by $\underline{A}$ and $\bar{A}$. Thus $A=[\underline{A}, \bar{A}]$. The intervals $A$ and $B$ are said to be equal if they are the same sets.

$$
\mathrm{A}=\mathrm{B} \text { if and only if } \underline{A}=\underline{B} \text { and } \bar{A}=\bar{B}
$$

Definition (2-1-2): ( Endpoint formulas of the arithmetic operations)

Let us find and operational way to add interval. Since

$$
\begin{array}{ll}
a \in A \text { means that } & \underline{A} \leq a \leq \bar{A} \\
b \in B \text { means that } & \underline{B} \leq b \leq \bar{B}
\end{array}
$$

We see by addition of inequalities that the numerical sum $a+b \epsilon A+B$ must

Satisfy

$$
\underline{\mathrm{A}}+\underline{\mathrm{B}} \leq \mathrm{a}+\mathrm{b} \leq \overline{\mathrm{A}}+\overline{\mathrm{B}}
$$

Hence

$$
\mathrm{A}+\mathrm{B}=[\underline{A}+\underline{B}, \bar{A}+\bar{B}]
$$

And

$$
-A=[-\bar{A},-\underline{A}]=\{a: a \in A\}
$$

For subtraction we add the inequalities

$$
\underline{A} \leq a \leq \bar{A} \text { and }-\underline{B} \leq-b \leq-\bar{B}
$$

To get

$$
\underline{A}-\bar{B} \leq a-b \leq \bar{A}-B
$$

It that

$$
A-B=[\underline{A}-\bar{B}, \bar{A}-\underline{B}]
$$

Defined product $A \times B$ of two interval $A$ and $B$. [10, 11, 12]

$$
A \times B=[\min s, \max s], \text { where } s=\{\underline{A} B, \underline{A} \bar{B}, \bar{A} \underline{B}, \overline{A B}\}
$$

A two-dimensional interval vector

$$
A=\left[A_{1}, A_{2}\right]=\left[\left[\underline{A}_{1}, \bar{A}_{1}\right],\left[\underline{A}_{2}, \bar{A}_{2}\right]\right]
$$


Can be represented as a rectangle in the $a_{1} a_{2}$-plans: It is the set of all point $\left(a_{1}, a_{2}\right)$ such that

$$
\underline{A_{1}} \leq \underline{a_{1}} \leq \overline{A_{1}} \quad, \quad \underline{A_{2}} \leq \underline{a_{2}} \leq \overline{A_{2}}
$$

Definition (2-1-3): The support of an interval number is given as $A=[\underline{A}, \bar{A}]$, an interval number is said to be positive if $O \leq \underline{A} \leq \bar{A}$. Similarly, an interval number is said to be negative if $\underline{A} \leq \bar{A}<0$.

The width and midpoints of an interval $A$ is defined and denoted by

$$
\omega(A)=\bar{A}-\underline{A} \quad \text { and } \quad m(A)=\frac{\bar{A}+\underline{A}}{2}
$$

\section{2-2: Inverse of an interval matrix [13].}

Definition (2-2-1):An interval matrix $\widetilde{A}$ is a matrix whose elements are interval numbers. An interval matrix $\widetilde{A}$ will be written as: $\tilde{A}=\left[\begin{array}{ccc}\tilde{a}_{11} & \cdots & \tilde{a}_{1 n} \\ \cdots & \cdots & \cdots \\ \tilde{a}_{n 1} & \cdots & \tilde{a}_{n n}\end{array}\right]=\left(\tilde{a}_{i j}\right) 1 \leq i \leq m, 1 \leq j \leq n$ where each $\widetilde{a}_{i j}=\left[\underline{a}_{i j}, \bar{a}_{i j}\right]$ or $\tilde{A}=[\underline{A}, \bar{A}]$ for some $\underline{A}, \bar{A}$ satisfying $\underline{A}<\bar{A}$. We use $R^{m \times n}$ to denote the set of all $(\mathrm{m} \times \mathrm{n})$ interval matrices. The midpoint (center) of an interval matrix $\widetilde{A}$ is the matrix of midpoints of its interval elements definedas $m(\tilde{A})=\left[\begin{array}{ccc}m\left(\tilde{a}_{11}\right) & \cdots & m\left(\tilde{a}_{1 n}\right) \\ \cdots & \cdots & \cdots \\ m\left(\tilde{a}_{n 1}\right) & \cdots & m\left(\tilde{a}_{n n}\right)\end{array}\right]$.

We introduce the following arithmetic operations on interval matrices. As with interval numbers, we define the arithmetic operations on interval matrices as follows. If $\tilde{A}, \tilde{B} \in R^{m \times n}, \tilde{X} \in R^{n}$ and $\tilde{\alpha} \in R$, then

$$
\begin{aligned}
& \text { (i). } \tilde{\alpha} \tilde{A}=\left(\tilde{\alpha} \tilde{a}_{i j}\right) \quad 1 \leq i \leq m, \quad 1 \leq j \leq n . \\
& \text { (ii). } \tilde{A}+\tilde{B}=\left(\tilde{a}_{i j}+\tilde{b}_{i j}\right) 1 \leq i \leq m, \quad 1 \leq j \leq n . \\
& \text { (iii). } \tilde{A}-\tilde{B}=\left\{\begin{array}{l}
\left(\tilde{a}_{i j}-\tilde{b}_{i j}\right) 1 \leq i \leq m, \quad 1 \leq j \leq n, \quad \text { if } \tilde{A} \neq \tilde{B} \\
\tilde{A}-\operatorname{dual}(\tilde{A})=\tilde{0}=0, \quad \text { if } \tilde{A}=\tilde{B}
\end{array}\right. \\
& \tilde{A}=[\underline{A}, \tilde{A}] \rightarrow \text { dual } \tilde{A}=\operatorname{dual}[\underline{A}, \bar{A}]=[\bar{A}, \underline{A}] . \\
& \text { (iv). } \tilde{A} \tilde{B}=\left(\sum_{k=1}^{n} \tilde{a}_{i k} \tilde{b}_{k j}\right) \quad 1 \leq i \leq m, 1 \leq j \leq n . \\
& \text { (v). } \tilde{A} \tilde{X}=\left(\sum_{j=1}^{n} \tilde{a}_{i j} \tilde{x}\right) \quad 1 \leq i \leq m .
\end{aligned}
$$

Definition (2-2-2): We define the determinant of a square interval matrix as in the case of real square matrix except that the determinant of an interval matrix is an interval number. 
That isdet $\tilde{A}=|\tilde{A}|=\sum \tilde{a}_{i j} \tilde{A}_{i j}$, where $\tilde{A}_{i j}$ is the cofactor of $\tilde{a}_{i j}$ with usual meaning.It is easy to see that most of the properties of determinants of classical matrices are hold good (up to equivalent) for the determinants of interval matrices under the modified interval arithmetic.

Definition (2- 2-3): A square interval matrix $\tilde{A}$ is said to be non singular or regular if $|\tilde{A}|$ is invertible (i.e, $0 \notin|\tilde{A}|$ ). Alternatively, a square interval matrix $\tilde{A}$ is said to be invertible if $|\tilde{A}|$ is invertible (i.e, $0 \notin|\tilde{A}|)$.

Example (2-2-3) :Let $\quad \tilde{A}=[\underline{A}, \bar{A}]=\left[\begin{array}{cc}{[1,2]} & {[3,4]} \\ {[-9,1]} & {[8,10]}\end{array}\right]$

Then $|\tilde{A}|=\left|\begin{array}{cc}{[1,2]} & {[3,4]} \\ {[-9,1]} & {[8,10]}\end{array}\right|=[1,2][8,10]-[-9.1][3,4]=[8,20]-[-36,4]=[4,56]$

We see that $0 \notin|\tilde{A}|=[4,56]$ and hence $|\tilde{A}|$ is invertible. So that $\tilde{A}$ is regular.

Definition (2-2-4): Let $\widetilde{A}$ be a square interval matrix. The adjointmatrix $\tilde{A}^{*}$ of $\tilde{A}$ is the transpose of the matrix of cofactors of the elements of $\tilde{A}$.

That is $\tilde{A}^{*}=\operatorname{adj}(\tilde{A})=\left(\tilde{b}_{i j}\right)$, where $\tilde{b}_{i j}=\left|A_{i j}\right|$, for all $i, j=1,2,3, \ldots, n$

Some of the solution of linear systems procedures are based an extension arithmetic .On the other hand, $[2,7,11,14]$.Consider

$$
\tilde{A} \tilde{X}=\tilde{B}
$$

Matrix $\tilde{A}$ in this equation represents the coefficient matrix. $\widetilde{X}$ vector is unknown and $\tilde{B}$ vector right-hand side.

\section{3-1: Calculating interval linear system ([14])}

In this section, some definitions will be introduced in order to help in calculating linear system. These are multivariate function and gradient.

Definition (3-1-1). Consider the $\mathrm{n} \times \mathrm{n}$ liner system of equations:

$$
\left\{\begin{array}{c}
\left(\tilde{a}_{11} \times \tilde{x}_{1}\right)+\left(\tilde{a}_{12} \times \tilde{x}_{2}\right)+\cdots+\left(\tilde{a}_{1 n} \times \tilde{x}_{n}\right)=\tilde{b}_{1} \\
\left(\tilde{a}_{21} \times \tilde{x}_{1}\right)+\left(\tilde{a}_{22} \times \tilde{x}_{2}\right)+\cdots+\left(\tilde{a}_{2 n} \times \tilde{x}_{n}\right)=\tilde{b}_{2} \\
\vdots \\
\left(\tilde{a}_{n 1} \times \tilde{x}_{1}\right)+\left(\tilde{a}_{n 2} \times \tilde{x}_{2}\right)+\cdots+\left(\tilde{a}_{n n} \times \tilde{x}_{n}\right)=\tilde{b}_{n}
\end{array}\right.
$$

The matrix form of the above equation is $\tilde{A} \tilde{x}=\tilde{b}$. Then for $2 \times 2$ matrix we have:

$$
\left[\begin{array}{ll}
\tilde{a}_{11} & \tilde{a}_{12} \\
\tilde{a}_{21} & \tilde{a}_{22}
\end{array}\right]\left[\begin{array}{l}
\tilde{x}_{1} \\
\tilde{x}_{2}
\end{array}\right]=\left[\begin{array}{l}
\tilde{b}_{1} \\
\tilde{b}_{2}
\end{array}\right](3)
$$

Where $\tilde{a}_{i j}=\left[\underline{a_{i j}}, \overline{a_{i j}}\right], \tilde{x}_{i}=\left[\underline{x}_{i}, \bar{x}_{i}\right], \tilde{b}_{i}=\left[\underline{b_{i}}, \overline{b_{i}}\right]$, for $i, j=1,2$.

Definition (3-1-2).[4]: In this section we find inversion approximately another method for solving the linear system of equations in crisp case $A x=b$, is Cramer's rule which states that each entry $x_{i}$ in the 
solution is a quotient of two determinants [15]. For solving interval linear systems (1) With this method, consider Eq, (2). Thus $x_{i}=\left(x_{1}, x_{2}, \ldots, x_{n}\right)$ is called the answer equation if the

$$
x_{i}=\frac{\operatorname{det}(\tilde{A}(i)}{\operatorname{det}(\tilde{A})} \quad i=1,2, \ldots, n,
$$

Where $\tilde{A}^{(i)}$ denotes the matrix obtained from $\tilde{A}$ by replacing its $i$ th column by $\tilde{b}$. Based on extension principle, Then $x_{i}=\left(\underline{x}_{i}, \bar{x}_{i}\right)$

Obviously:

$$
\begin{aligned}
& \underline{x}_{i}=\left\{\min \frac{\operatorname{det}\left(A^{(i)}\right)}{\operatorname{det}(A)} \mid a_{i j} \leq a_{i j} \leq \bar{a}_{i j}, \quad \underline{b}_{i j} \leq b_{i j} \leq \bar{b}_{i j}\right\} \\
& \bar{x}_{i}=\left\{\max \frac{\operatorname{det}\left(A^{(i)}\right)}{\operatorname{det}(A)} \mid a_{i j} \leq a_{i j} \leq \bar{a}_{i j}, \quad \underline{b}_{i j} \leq b_{i j} \leq \bar{b}_{i j}\right\}
\end{aligned}
$$

$$
x_{i}=f_{i}\left(a_{11}, a_{12} \ldots, a_{n 1}, a_{n n}, b_{1}, \ldots b_{n}\right), \quad i,=1,2, \ldots, n
$$

Definition (3-1-3): (Multivariate function.)

If the functions range is a sub- set of real numbers and is called the scalar function. If the domain and range of a function of $R^{n}$ and $R^{m}$ are non-empty subsets of vector $\mathrm{n}$, then the function will be called the multivariate vector function. Then $f: A \subset R^{n} \rightarrow R$

If $f: A \subset R^{n} \rightarrow R$ is a multivariate function then for every $x \in A, f(x) \in R$. Thus we have

$$
f(x)=f\left(x_{1}, x_{2}, \ldots, x_{n}\right)
$$

Theorem (3-1-1): $f: A \subset R^{n} \rightarrow R, f(x)=f\left(x_{1}, x_{2}, \ldots, x_{n}\right)$ is continuous forc $\epsilon R^{n}$ if and only if the entire components are continuous at this point. A special case of multivariate functions has been considered.

Definition (3-1-4): If there is the partial function of $f: A \rightarrow R$ at the point $x_{0} \in A$, then the vector, will be called the gradient of $f$ at the point $x_{0}$, and it will be represented by the symbol $\nabla f\left(x_{0}\right)$.

$$
\nabla f\left(x_{0}\right)=\left(\frac{\partial f}{\partial x_{1}}\left(x_{0}\right), \ldots, \frac{\partial f}{\partial x_{n}}\left(x_{0}\right)\right)
$$

If the function $f: R^{n} \rightarrow R$ in the neighborhood of the point $x_{0}=\left(x_{1}{ }^{(0)}, x_{2}{ }^{(0)}, \ldots, x_{n}{ }^{(0)}\right)$ ispartial, and the partial derivatives are continuous at the point $x_{0}$, then the derivative of $f$ at the point $x_{0}$ and $u$ will be as follows:

$$
D_{u} f(x)=\nabla f(x) \cdot u
$$

The negative derivative of $f$ at the point $x_{0}$ and the gradient vector inner product of $u$ is the unit vector $u$.

The maximum value of the derivative at each point is the direction of the gradient and the minimum value of the derivative at each point can be obtained in the opposite direction of the gradient. 


\section{3-2: Implementation. [6]}

The derivative of $f$ at (an interior point of its domain) $x$, denoted by $\mathrm{f}^{\prime}(x)$, and defined by

$$
f^{\prime}(x)=\lim _{x_{n} \rightarrow x} \frac{f(x)-f\left(x_{n}\right)}{x-x_{n}}
$$

Let us consider a differentiable function $f: R \rightarrow R$. The derivative offat (an interior point of its domain) $x$ is denoted by $f^{\prime}(x)$. If $f^{\prime}(x)>0$ then we say that $f$ is increasing at $x$, if $f^{\prime}(x)<0$ then we say that $f$ is decreasing at $x$, if $f^{\prime}(x)=0$ then $f$ can have a local maximum, minimum or inflexion point at $x$.

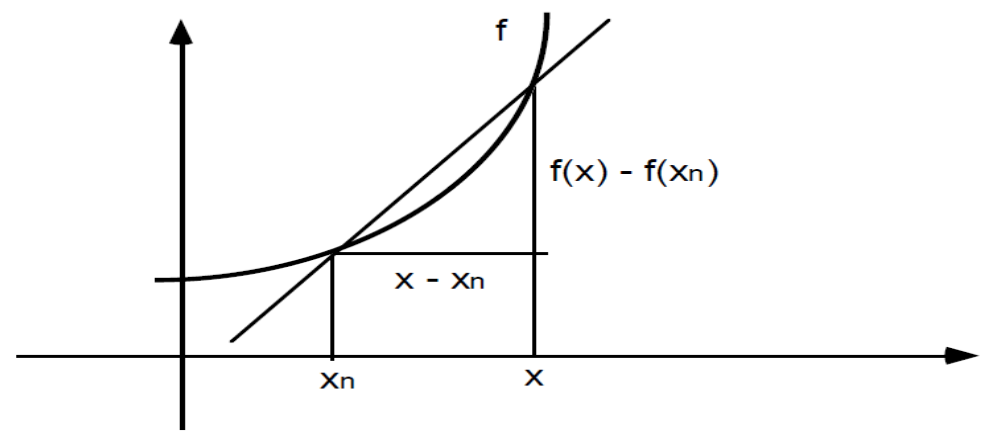

Figure 3-1 Derivative of function $f$.

A differentiable function is always increasing in the direction of its derivative, and decreasing in the opposite direction. It means that if we want to find one of the local minima of a function $f$ starting from a point $x^{\circ}$ then we should search for a second candidate in the right-hand side of $x^{\circ}$ if $f^{\prime}\left(x^{\circ}\right)<0$ (when $f$ is decreasing at $x^{\circ}$ ) and in the left-hand side of $x^{\circ}$ if $f^{\prime}\left(x^{\circ}\right)>0$ (when $f$ increasing at $x^{\circ}$ ). The equation for the line crossing the point $\left(x^{\circ} ; f^{\prime}\left(x^{\circ}\right)\right)$ is given by

That is

$$
\frac{y-f\left(x^{\circ}\right)}{x-x^{\circ}}=f^{\prime}\left(x^{\circ}\right)
$$

$$
y=f\left(x^{\circ}\right)+\left(x-x^{\circ}\right) f^{\prime}\left(x^{\circ}\right)
$$

The next approximation, denoted by $x^{1}$, is a solution to the equation

Which is

$$
0=f\left(x^{\circ}\right)+\left(x-x^{\circ}\right) f^{\prime}\left(x^{\circ}\right)
$$

$$
x^{l}=x^{\circ}-\frac{f\left(x^{\circ}\right)}{f^{\prime}\left(x^{\circ}\right)}
$$

This idea can be applied successively, that is

$$
x^{(n+1)}=x^{(n)}-\frac{f\left(x^{(n)}\right)}{f^{\prime}\left(x^{(n)}\right)}
$$




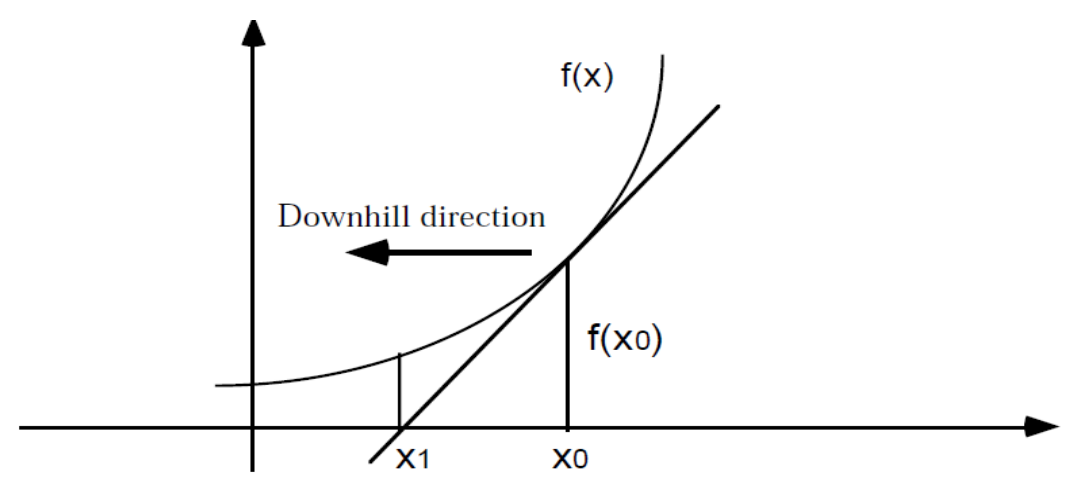

Figure 3-2 The downhill direction is negative at $x_{0}$

The above procedure is a typical descent method. In a descent method the next iteration $x^{(n+1)}$ should satisfy the following property

$$
f\left(x^{(n+1)}\right)<f\left(x^{(n)}\right)
$$

I.e. the value of $f$ at $x^{(n+1)}$ is smaller than its previous value at $x^{(n)}$.

In error correction learning procedure, each iteration of a descent method calculates the downhill direction (opposite of the direction of the derivative) at $x^{(n)}$ which means that for a sufficiently small $\mu>0$ the inequality

should hold, and we let $x^{(n+1)}$ be the vector

$$
f\left(x^{(n)}-\mu f^{\prime}(x)^{(n)}\right)<f(x)^{(n)}
$$

$$
x^{(n+1)}=x^{(n)}-\mu f^{\prime}\left(x^{(n)}\right)
$$

Let $f: R^{n} \rightarrow R$ be a real-valued function. In a descent method, whatever is the next iteration, $x^{(n+1)}$, it should satisfy the property

$$
f\left(x^{(n+1)}\right)<f\left(x^{(n)}\right)
$$

I.e. the value of $f$ at $x^{(n+1)}$ is smaller than its value at previous approximation $x^{(n)}$. Each iteration of a descent method calculates a downhill direction (opposite of the direction of the derivative) at $(x)^{(n)}$ which means that for a sufficiently small $\mu>0$ the inequality

Should hold, and we let $x^{(n+1)}$ be the vector

$$
f\left(x^{(n)}-\mu f^{\prime}\left(x^{(n)}\right)<f\left(x^{(n)}\right)\right.
$$

$$
x^{(n+1)}=x^{(n)}-\mu f^{\prime}\left(x^{(n)}\right)
$$

The $\left(x_{i}\right)$ is function of $\left(a_{11}, a_{12} \ldots, a_{1 n}, \ldots, a_{n 1}, \ldots, a_{n n}, b_{1}, \ldots, b_{n}\right)$ i.e

$$
x_{i}=f_{i}\left(a_{11}, a_{12} \ldots, a_{1 n}, \ldots, a_{n 1}, \ldots, a_{n n}, b_{1}, \ldots, b_{n}\right), \quad i,=1,2, \ldots, n
$$

The goal, then, is to minimize this function. It turns out, if the output functions are differentiable, that this problem has a simple solution: namely, we can assign a particular unit blame in proportion to the degree to which changes in that unit's activity lead to changes in the error. That is, we change the weights of the system in proportion to the derivative of the error with respect to the weights. The rule for changing weights following presentation of input/output pair $\left(x_{1}{ }^{(n)}, x_{2}{ }^{(n)}\right)$ is given by the gradient descent method, i.e. we minimize the quadratic error function by using the following iteration process: 


$$
\begin{aligned}
\left(a_{11}, a_{12} \ldots, a_{1 n}, \ldots, a_{n 1}, \ldots, a_{n n}, b_{1}, \ldots, b_{n}\right)^{(m+1)} & \\
& =\left(a_{11}, a_{12} \ldots, a_{1 n}, \ldots, a_{n 1}, \ldots, a_{n n}, b_{1}, \ldots, b_{n}\right)^{(m)} \\
& -\mu\left(\frac{\partial x}{\partial a_{11}}, \frac{\partial x}{\partial a_{12}}, \ldots, \frac{\partial x}{\partial a_{n n}}, \frac{\partial x}{\partial b_{1}}, \ldots, \frac{\partial x}{\partial b_{n}}\right)
\end{aligned}
$$

\subsection{Summary}

- Step 1: The initial value is ${a_{i j}}^{(0)}$, which is positioned in the interval of $\left[\underline{a}_{i j}, \bar{a}_{i j}\right]$ and usually consider as the ( midpoint), It is also assumed that $\mu>0$

- Step 2: We compute the gradient with regard to new variables.

$$
\nabla x_{i}=\left(\frac{\partial x_{i}}{\partial a_{11}}, \frac{\partial x_{i}}{\partial a_{12}}, \ldots, \frac{\partial x_{i}}{\partial a_{n n}}, \frac{\partial x_{i}}{\partial b_{1}}, \ldots, \frac{\partial x_{i}}{\partial b_{n}}\right) \quad i=1, \ldots, n
$$

- Step 3: New matrix elements are follows:

$$
\begin{aligned}
\left(a_{11}, a_{12} \ldots, a_{1 n}, \ldots, a_{n 1}, \ldots, a_{n n}, b_{1}, \ldots, b_{n}\right)^{(m+1)} & =\left(a_{11}, a_{12} \ldots, a_{1 n}, \ldots, a_{n 1}, \ldots, a_{n n}, b_{1}, \ldots, b_{n}\right)^{(m)} \\
& -\mu\left(\frac{\partial x}{\partial a_{11}}, \frac{\partial x}{\partial a_{12}}, \ldots, \frac{\partial x}{\partial a_{n n}}, \frac{\partial x}{\partial b_{1}}, \ldots, \frac{\partial x}{\partial b_{n}}\right)
\end{aligned}
$$

- Step 4: If $\left(x_{i}^{(n+1)}\right)<\left(x_{i}^{(n)}\right)$ we continue the training by going back to Step 2, otherwise we go to Step 5 .

- Step 5: minimum values of the unknown quantity $x_{i}$ is the step before.

For simplicity: we will consider the square matrix which consists of interval numbers is and $x=\left(x_{1}, x_{2}\right)$.

\section{Numerical examples}

It this section one example is given in order to illustrate the proposed method. Let $\tilde{A}$ be $2 \times 2$ matrix which is given as follows and solve it using Cramer's rule:

$$
\left[\begin{array}{ll}
{[3,4]} & {[1,2]} \\
{[0,1]} & {[7,8]}
\end{array}\right]\left[\begin{array}{l}
{[\underline{x}, \bar{x}]} \\
{[\underline{y}, \bar{y}]}
\end{array}\right]=\left[\begin{array}{c}
{[2,4]} \\
{[-1,1]}
\end{array}\right]
$$

Due to (4):

$$
\begin{aligned}
x_{i} & =f_{i}\left(a_{11}, a_{12}, a_{21}, a_{22}, b_{1}, b_{2}\right) \\
\frac{\partial x_{1}}{\partial a_{11}} & =\frac{a_{22}\left(b_{2} a_{12}-a_{22} b_{1}\right)}{\left(a_{11} a_{22}-a_{12} a_{21}\right)^{2}} \frac{\partial x_{1}}{\partial a_{12}}=\frac{a_{22}\left(a_{21} b_{1}-b_{2} a_{11}\right)}{\left(a_{11} a_{22}-a_{12} a_{21}\right)^{2}} \\
\frac{\partial x_{1}}{\partial a_{21}} & =\frac{a_{12}\left(b_{1} a_{22}-a_{12} b_{2}\right)}{\left(a_{11} a_{22}-a_{12} a_{21}\right)} \frac{\partial x_{1}}{\partial a_{22}}=\frac{a_{12}\left(a_{11} b_{2}-a_{21} b_{1}\right)}{\left(a_{11} a_{22}-a_{12} a_{21}\right)^{2}} \\
\frac{\partial x_{1}}{\partial b_{1}} & =\frac{a_{22}}{a_{11} a_{22}-a_{12} a_{21}} \frac{\partial x_{1}}{\partial b_{2}}=\frac{-a_{12}}{a_{11} a_{22}-a_{12} a_{21}}
\end{aligned}
$$

Similarly $x_{2}$ can be obtains with respect to its variables. 
Step 1:

$$
\left[\begin{array}{cc}
3.5 & 1.5 \\
0.50 & 7.5
\end{array}\right]\left[\begin{array}{l}
x_{1}{ }^{(1)} \\
x_{2}{ }^{(1)}
\end{array}\right]=\left[\begin{array}{l}
3 \\
0
\end{array}\right]
$$

We solve the system:

$$
\begin{gathered}
x_{1}{ }^{(1)}=0.8823 \quad, \quad x_{2}{ }^{(1)}=-0.058 \\
\frac{\partial x}{\partial a_{11}}=-0.2595 \quad \frac{\partial x}{\partial a_{12}}=0.0173 \quad \frac{\partial x}{\partial a_{21}}=0.0519 \quad \frac{\partial x}{\partial a_{22}}=-0.0035 \quad \frac{\partial x}{\partial b_{1}}=0.2941 \\
\frac{\partial x}{\partial b_{2}}=-0.0588
\end{gathered}
$$

Assuming $\mu=0.05$ we have

Step 2:

$$
a_{i j}^{(2)}=a_{i j}^{(1)}-0.05(\nabla f)
$$

$a_{i j}{ }^{(2)}=(3.5,1.5,0.50,7.5,3,0)-0.05(-0.2595,0.0173,0.0519,-0.0035,0.2941,-0.0588)$

$=(3.5130,1.4991,0.4974,7.5002,2.9853,0.0029)$

$$
\begin{gathered}
{\left[\begin{array}{ll}
3.5130 & 1.4991 \\
0.4974 & 7.5002
\end{array}\right]\left[\begin{array}{l}
x_{1}(2) \\
x_{2}(2)
\end{array}\right]=\left[\begin{array}{l}
2.9853 \\
0.0029
\end{array}\right]} \\
x_{1}{ }^{(2)}=.8744, \quad, \quad x_{2}{ }^{(2)}=0.0576
\end{gathered}
$$

Step 43:

$$
\begin{gathered}
\frac{\partial x}{\partial a_{11}}=-0.1635 \frac{\partial x}{\partial a_{12}}=0.0052 \frac{\partial x}{\partial a_{21}}=0.0322 \quad \frac{\partial x}{\partial a_{22}}=-0.0010 \quad \frac{\partial x}{\partial b_{1}}=0.2603 \\
\frac{\partial x}{\partial b_{2}}=-0.0513 \\
a_{i j}{ }^{(43)}=a_{i j}{ }^{(42)}-0.05(\nabla f) \\
a_{i j}{ }^{(43)}=(3.9240,1.4782, .4159,7.5043,2.4350,0.1120) \\
-0.05(-0.1635,0.0052,0.0322,-0.0010,0.2603,-0.0513) \\
=(3.9322,1.4779,0.4143,7.5044,2.4220,0.1146)
\end{gathered}
$$




$$
\begin{gathered}
{\left[\begin{array}{ll}
3.9322 & 1.4779 \\
0.4143 & 7.5044
\end{array}\right]\left[\begin{array}{l}
x_{1}{ }^{(43)} \\
x_{2}{ }^{(43)}
\end{array}\right]=\left[\begin{array}{l}
2.4220 \\
0.1146
\end{array}\right]} \\
x_{1}^{(43)}=0.6231 \quad, \quad x_{2}{ }^{(43)}=-0.0191
\end{gathered}
$$

And for $\bar{x}_{i}$ we have:

Step 1:

$$
\left[\begin{array}{cc}
3.5 & 1.5 \\
0.50 & 7.5
\end{array}\right]\left[\begin{array}{l}
x_{1}{ }^{(1)} \\
x_{2}{ }^{(1)}
\end{array}\right]=\left[\begin{array}{l}
3 \\
0
\end{array}\right]
$$

We solve the system:

$$
\begin{gathered}
x_{1}{ }^{(1)}=0.8823 \quad, \quad x_{1}{ }^{(1)}=-0.058 \\
\frac{\partial x}{\partial a_{11}}=-0.2595 \quad \frac{\partial x}{\partial a_{12}}=0.0173 \quad \frac{\partial x}{\partial a_{21}}=0.0519 \quad \frac{\partial x}{\partial a_{22}}=-0.0035 \quad \frac{\partial x}{\partial b_{1}}=0.2941 \\
\frac{\partial x}{\partial b_{2}}=-0.0588
\end{gathered}
$$

Assuming $\mu=0.05$ we have

Step 2:

$$
a_{i j}^{(2)}=a_{i j}^{(1)}+0.05(\nabla f)
$$

$a_{i j}{ }^{(2)}=(3.5,1.5,0.50,7.5,3,0)+0.05(-0.2595,0.0173,0.0519,-0.0035,0.2941,-0.0588)$

$=(3.4870,1.5009, .5026,7.4998,3.0147,-0.0029)$

$$
\begin{gathered}
{\left[\begin{array}{ll}
3.4870 & 1.5009 \\
0.5026 & 7.7998
\end{array}\right]\left[\begin{array}{l}
x_{1}^{(2)} \\
x_{2}(2)
\end{array}\right]=\left[\begin{array}{c}
3.0147 \\
-0.0029
\end{array}\right]} \\
x_{1}{ }^{(2)}=0.8904 \quad, \quad x_{2}{ }^{(2)}=-0.0601 \\
\vdots
\end{gathered}
$$

Step 31:

$$
\begin{aligned}
& \frac{\partial x}{\partial a_{11}}=-0.4097 \quad \frac{\partial x}{\partial a_{12}}=0.0367 \quad \frac{\partial x}{\partial a_{21}}=0.0840 \quad \frac{\partial x}{\partial a_{22}}=-0.0075 \quad \frac{\partial x}{\partial b_{1}}=0.3434 \\
& \frac{\partial x}{\partial b_{2}}=-0.0704 \\
& a_{i j}^{(31)}=a_{i j}^{(30)}+0.05(\nabla f)
\end{aligned}
$$




$$
\begin{aligned}
& a_{i j}{ }^{(31)}=(3.0343,1.5366,0.5942,7.4926,3.4564,-0.0923) \\
& +.05(-0.4097,0.0367,0.0840,-0.0075,0.3434,-0.0704) \\
& =(3.0138,1.5384,0.05984,7.4922,3.4736,-0.0958) \\
& {\left[\begin{array}{ll}
3.0138 & 1.5384 \\
0.5984 & 7.4922
\end{array}\right]\left[\begin{array}{l}
x_{1}(31) \\
x_{2}(31)
\end{array}\right]=\left[\begin{array}{c}
3.4736 \\
-0.0958
\end{array}\right]} \\
& x_{1}^{(31)}=1.2083 \quad, \quad x_{2}{ }^{(31)}=-0.1093
\end{aligned}
$$

Thus consider the value of $x_{1}$ as follows.

$$
\left[\underline{x_{1}}, \overline{x_{1}}\right]=(0.6231,1.2083)
$$

And similarly for $x_{2}$ also obtains wehave:

$$
\left[\underline{x_{2}}, \overline{x_{2}}\right]=(-0.2075,-0.0520)
$$

By using the same notion $2 \times 2$ case can be easily extended to $n \times n$ case.

\section{Conclusion}

In this paper, a method is proposed to calculate the interval linear systems. To solve this equation system, some definitions such interval matrix are introduced. According to the mentioned definitions, Cramer's rule and gradient vector are also defined and applied in order to calculate the interval linear systems. It should be carefully considered that in order to calculate the interval linear systems by using our proposed method. We represent an efficient algorithm for finding the interval solution of the interval linear system. This algorithm applies optimization problem based on gradient vector in order to obtain the lower bound and upper bound of the interval solution.

\section{References}

[1] M. Alperbazaran, Calculation fuzzy inverse matrix using fuzzy linear equation system, Applied Soft Computing 12(2012)1810-1813

[2] G. Alefeld and J. Herzberger, Introduction to Interval Computations, Academic Press, New York 1983.

[3] T. Allahviranloo, Successive over relaxation iterative method for fuzzy system of linear equation, Applied Mathematics and Computation 162 (2005) 189-196.

[4]M. Dehngan, B. Hashemi, Iterative solution of fuzzy linear systems, Applied Mathematics and Computation 175 (2006) 645-674.

[5] D. Dubois, H. Prade, Systems of linear fuzzy constraints, International Journal of Systems Science 9 (1978) 613-626.

[6] R, Fuller. Neural Fuzzy Systems .Donner visiting professor Abo Akademi university ISBN 951-650624-0, ISSN 0358-5654.

[7] K. Ganesan and P. Veeramani, On Arithmetic Operations of Interval Numbers, International Journal of Uncertainty, Fuzziness and Knowledge- Based Systems, 13 (6) (2005), 619 - 631.

[8] K. Ganesan, On Some Properties of Interval Matrices, International Journal of Computational and Mathematical Sciences, 1 (2) (2007), 92-99 
[9] S.Markov, Computation of algebraic solution to interval system via system of coordinates, Scientific computing, Validated Numerics, Interval methods, Eds. W. Kraemer, J. Wolff von Gudenberg, Kluwer, (2001),103-114.

[10] E.Moor,R Baker Kear foot, Michael.J. Cloud, Introduction to interval Analysis .(studies in Applied Mathematics, SILM , philaderphia,1979 PA,19104 -2688 USA.

[11] Sukanta Nayak, S. Chakraverty, A new Approch to solve Fuzzy system of linear Equation, contents list available at tjmcs, journal of mathematics and computer science 7(2013), 205-212.

[12] S.H.Nasseri, F.Zahmatkesh, Huang method for solving full fuzzy linear system, The journal of Mathematics and Computer Science, 1 (2010),1-5.

[13] T. Nirmal. D.Datta. H.S. Kushwaha. K. Ganesan. Invers interval matrix: E new approach Applied Mathematics, Sciences Vol.5, 201, no 13,607-624

[14] E. R. Hansen and R. R. Smith, Interval arithmetic in matrix computations, Part 2, SIAM. Journal of Numerical Analysis, 4 (1967), 1 - 9.

[15] D.S. Watkins, Fundamentals of Matrix Computations, Wiley-Interscience Pub., New York, 2002. 